\title{
Framework for Quality Metrics in Mobile-Wireless Information Systems ${ }^{1}$
}

\author{
Ruti Gafni \\ Bar Ilan University, Ramat Gan, Israel
}

rutiga@openu.ac.il

\begin{abstract}
Mobile and wireless devices and networks enable "any place, any time" use of information systems, providing advantages, such as productivity enhancement, flexibility, service improvements and information accuracy. These benefits can be nullified by using systems of insufficient quality. Mobile-wireless information systems face new kinds of problems: narrow bands, small devices, tiny screens, and diversity of users and devices. Information systems quality cannot be measured only by software faults absence; it must be broader, including characteristics to cover all aspects, life-cycle phases, and viewpoints. This research develops a methodology to define and quantify the quality components of such systems, merging two fields together: mobile-wireless applications and standards for product quality assurance. The research has developed a list of questions from which new quality metrics were defined and empirically validated, extending the ISO/IEC 9126 quality standard for mobile-wireless information systems. The paper describes the metrics development process and presents examples of metrics.
\end{abstract}

Keywords: information systems, mobile, wireless, quality, software engineering.

${ }^{1}$ This article is based on the author's PhD dissertation conducted in the Business Administration School at Bar-Ilan University, Israel, supervised by Dr. Moshe Mor and Dr. David Schwartz.

\section{Introduction}

Technology improvements enable the building of information systems which can be used "any place, any time", through mobile and wireless devices and networks. Mobile-wireless information systems can create benefits for organizations; e.g., productivity enhancement, processes and procedures flexibility, customer services improvement and information accuracy for decision makers, which together emphasize competitive strategy, lower operation costs, and improved processes. However, these benefits can be nullified by using non quality information systems. In order to use these systems successfully, they need to be of good quality (Terho, 2002). Quality is a multi-dimension concept which includes a multitude of characteristics. Several attempts have been made to examine the information systems quality nature, to define quality components and

Material published as part of this publication, either on-line or in print, is copyrighted by the Informing Science Institute. Permission to make digital or paper copy of part or all of these works for personal or classroom use is granted without fee provided that the copies are not made or distributed for profit or commercial advantage AND that copies 1) bear this notice in full and 2) give the full citation on the first page. It is permissible to abstract these works so long as credit is given. To copy in all other cases or to republish or to post on a server or to redistribute to lists requires specific permission and payment of a fee. Contact Publisher@InformingScience.org to request redistribution permission. to find systematic ways to measure them (ISO/IEC 9126-1, 2001; Kan, 2002; Pandian, 2004). Many information systems fail due to poor quality. Research has been focused on traditional (Kan, 2002) and internet information systems (Calero, Ruiz, \& Piattini, 2004; Covella \& Olsina, 2006). However, mobilewireless information systems face new kinds of problems, such as narrow bands, lack of coverage, devices with 
small memory and screens which cannot display large amount of data, and diversity of users and devices. The special mobile-wireless information systems characteristics require a different quality definition.

This research uses software engineering to define the mobile-wireless information systems quality components and develops a methodology to quantify these components, in order to enable the evaluation, comparison, and analysis of mobile-wireless information systems quality.

The research process, as shown in Figure 1, consists of several phases: (1) mobile-wireless information systems examination to detect special quality problems and risks that result from the architectures and protocols of such systems; (2) choice of ISO/IEC 9126 quality characteristics affected by mobility and wirelessness; (3) definition of objects and metrics to allow objective measurement of mobile-wireless information systems quality; (4) metrics theoretical and empirical validation.

This paper focuses on the effects of mobile-wireless information systems on quality characteristics and introduces a list of questions from which new metrics are defined and mapped according to different viewpoints. In addition, two of the new metrics are described.

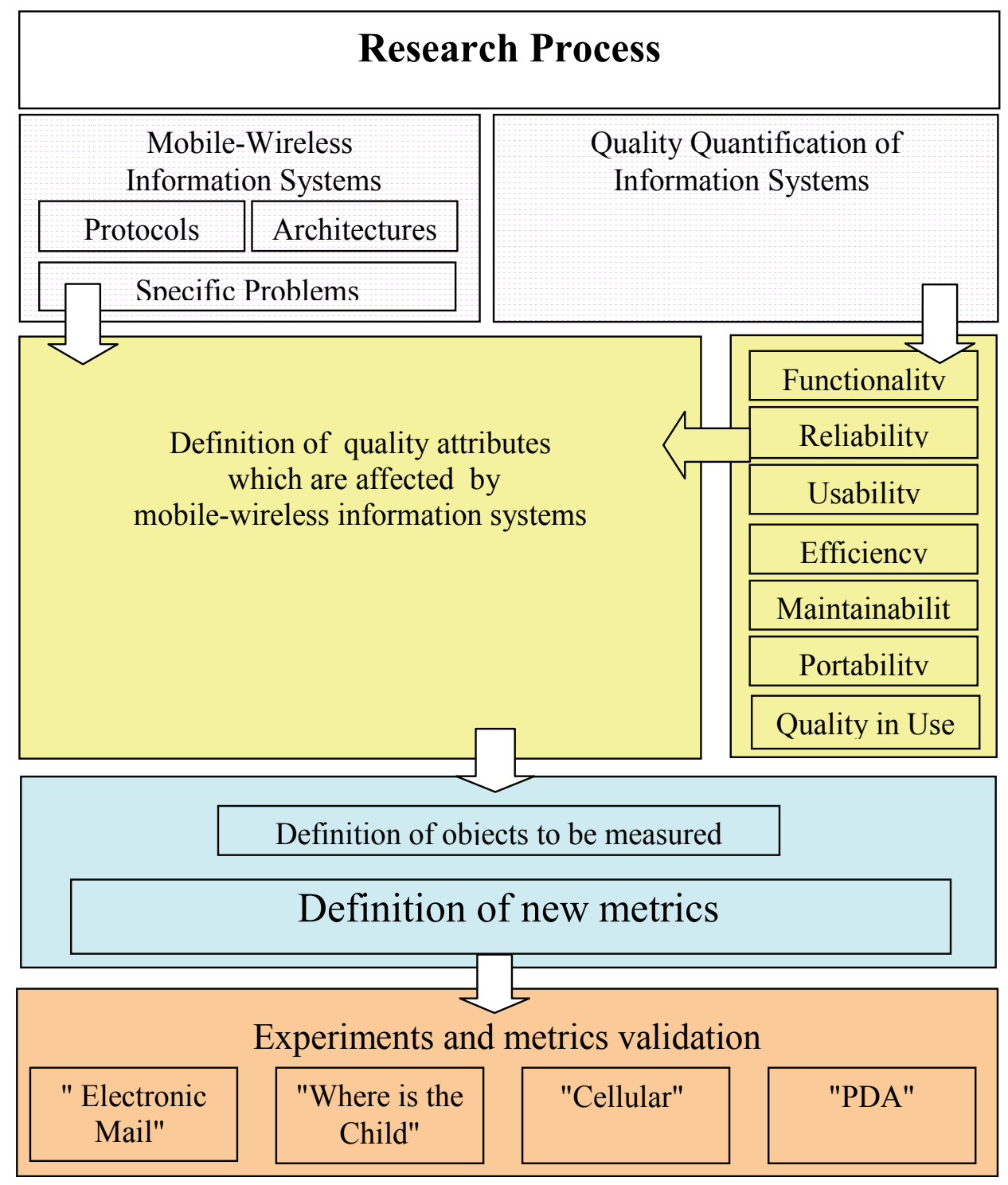

Figure 1 - The research process 


\section{Mobile-Wireless Information Systems}

Schiller (2000) describes two mobility dimensions: user mobility, which allows connection to the system from different geographical sites, and device mobility, which enables mobility of both user and system, such as laptops, personal digital assistants (PDA), or cellular phones. This research focuses on mobile-wireless information systems, which allow users to be connected any time at any place.

There were unsuccessful attempts to develop mobile-wireless information systems at the beginning of the millennium, since technology, devices and infrastructures were immature. This situation changed during 2003, and analysts agreed that these kinds of systems will grow and their market share will expand (Lau, 2006; Lee, 2003). The major reasons are emergence of third generation wireless networks (UMTS - Universal Mobile Telecommunications System, CDMA2000 $1 \mathrm{x}$ - Code division multiple access, and GPRS - General Packet Radio Service), their coverage expansion, and development of smart mobile devices.

\section{Applications Based on Mobile-Wireless Information Systems}

There are two classifications of mobile-wireless information systems: horizontal and vertical (Stafford \& Gillenson, 2003). Horizontal applications are general, adaptable to a wide range of users and organizations, e.g.: e-mail, browsers, and file transfer applications. Vertical applications are specific to a type of users or organizations, for example: financial applications, such as money transfer, stock exchange and information inquiry; marketing and advertising applications according to the actual user position, i.e., pushing coupons to stores and information about sales nearby; emergency applications to check real-time information from government and medical databases and utility companies applications used by technicians and meter readers.

There are several advantages of using these systems in the place and time of the event occurrence, in particular, productivity enhancement, resource allocation flexibility, competitive advantages, service improvements, and information accuracy (Malladi \& Agrawal, 2002).

\section{Problems in Mobile-Wireless Information Systems}

Mobile-wireless information systems face some unique problems originating from the mobile devices. First, these devices have small memories, short battery life, and limited calculation and computation capabilities. Second, there is a wide variety of devices, possessing different characteristics, and the application must be adaptable to all of them. Third, the use of the devices is uncomfortable because of their size, tiny screens, low resolution, and small keyboards that are difficult to operate. Fourth, security problems can arise when devices are lost, due to possible unauthorized access to sensitive data.

The network causes other problems, including: limited bandwidth, inconsistent connection stability, transfer delays, and varied standards and protocols, some with high overhead, decreasing the performance level. Moreover, when users operate the system during mobility, the connection point to the network can change, obstacles can disturb, causing temporary disconnections, interruptions, or disturbances. Besides, security, privacy, and confidentiality are very important issues (Di Pietro \& Mancini, 2003; Herzberg, 2003).

These problems intensify when the information system is targeted for a wide audience, where the users are faceless and there is neither user training nor implementation, but they are significant also in closed organizational information systems. 


\section{Information Systems Quality Measuring}

Measuring creates a quantitative description of processes and products which allows behavior comprehension. This knowledge enables the selection of tools and techniques to control and improve processes, products, and resources. People at dissimilar tasks: developers, managers, users, etc. have different viewpoints on quality. Information systems quality cannot be measured only by the absence of software faults; it must be broader, including characteristics to cover all aspects, life-cycle phases, and viewpoints.

Several standards and frames have been defined for information systems development process measuring. The most recognized are CMMI (Capability Maturity Model Integration) (Chrissis, Konrad, \& Shrum, 2006), ISO9000:2000 (ISO9000, 2000) with its ISO90003 (ISO90003, 2004) guidelines for software and ISO/IEC 15504 (ISO/IEC 15504, 2004, 2006), formerly known as SPICE (Software Process Improvement and Capability Determination) which integrates CMMI and ISO90003.

Product quality measuring is complicated, since there is no consensus on quality meaning. The most recent model, defined by the International Organization for Standardization and the International Electrotechnical Commission, is ISO/IEC 9126 (ISO/IEC 9126-1, 2001) standard that decomposes quality into several characteristics, further divided into sub-characteristics. This standard defines internal metrics to be measured without having to operate the system, external metrics to be measured while testing or executing the system, and quality in use, which is the system quality from the user's viewpoint.

\section{What Do We Need To Measure?}

Mobile-wireless information systems must be measured on the basis of traditional systems metrics, e.g. ease of maintainability, minimum complexity, lack of faults, mean time between failures (MTBF), etc., which are the fundamental metrics for all information systems. Furthermore, they must be measured according to internet systems metrics, e.g. no broken links, ease of navigation, etc. In addition, they need to be measured with special targeted mobile-wireless metrics (Spriestersbach \& Springer, 2004).

In this research, an additional questions list, specific for mobile-wireless information systems, was developed using Goal-Question-Metric approach (Basili \& Rombach, 1988), which constituted the basis for quantitative metrics definition. The mobility and wireless influence on ISO/IEC 9126 quality characteristics are described below, followed by the relevant questions.

\section{Functionality}

Functionality (ISO/IEC 9126-1, 2001) includes four sub-characteristics: suitability, accuracy, interoperability, and security. In location aware applications, using the automatic user location can alleviate the input operation, improving suitability. However, the user location can change during the transaction performance, causing incorrect output and affecting the accuracy. The application may need to access data at different servers and interact with one or more specified systems. The application must use standard protocols in order to perform this correctly and support interoperability. Security is affected in different ways, for example: loss or theft of the mobile device, exposure of the messages in the network to unauthorized access, and privacy loss because of location aware functions. The mobile-wireless information systems quality questions that need to be quantified are: 
- Does the application use location aware functions where applicable?

- Is the location aware functions output updated according to the user's position change during the transaction operation?

- Does the application apply user profile in order to adapt the output to user and device?

- Does the application use standard protocols and interfaces?

- Does the application include mechanisms of authentication, encryption, authorization, and confidence?

\section{Reliability}

Reliability (ISO/IEC 9126-1, 2001) includes the following sub-characteristics: maturity, fault tolerance, and recoverability. During mobility, network problems, hiding obstacles, and hopping between antennas may disturb and interrupt communication. Thus, the system must be fault tolerant to maintain a specified level of performance, and, when connection is suspended, the system needs to re-establish the communication and recover the data directly affected, supporting recoverability.

- To what degree does the mobility cause interruptions?

- Does the application use cache memory efficiently to avoid loss of data?

- Does the transaction interruption damage data?

\section{Usability}

Usability (ISO/IEC 9126-1, 2001) includes the following sub-characteristics: understandability, learnability, operability, and attractiveness. Usability is one of the most important characteristics when targeting systems to wide audiences, which need to operate an intuitive system without direct training and support. Mobile users may not be able to concentrate on the system use, so the application should not be complicated, the input must be easy to insert, intuitive, and simplified by using location aware functions (Terrenghi, Kronen, \& Valle, 2005). The operability subcharacteristic is affected mainly by the mobile device attributes: screen size, keyboard or numeric pad, etc., which restrict input and output interaction possibilities. Moreover, the noisy surroundings (when operating the system while on a street or public environments) may distract the user and cause input errors, inaccuracy, and slowness. The ability to reach the relevant data "any place any time" enlarges the system attractiveness.

- To what degree is the screen over-loaded and diminishes the application understandability?

- Are there specific menus for each possible operation?

- Are the buttons which operate each option clear enough?

- Is the help function for tasks easy to find?

- Is the application configurable according to user and device?

- Do the input fields have default values or choices instead of textual input in order to minimize errors?

- Does the system use location aware functions in order to minimize inputs?

- Is the length and format of the outputs optimized to screen size? 


\section{Efficiency}

Efficiency (ISO/IEC 9126-1, 2001) includes the time behavior and resource utilization subcharacteristics. Time behavior sub-characteristic is very important in the wireless environment because the price of each minute of data transferring is very high, and the users will avoid expensive systems. Moreover, users are accustomed to speedy systems, so slowness will not be accepted. Mobile devices include small memory and low processing resources, so applications must be aware of these restrictions and optimize resource utilization.

- Is the transaction execution time minimal?

- Does the application utilize the cache memory?

- Is the size of the application stored in the device proportional to the memory size?

- Is the size of the help system stored in the device proportional to the memory size and the application size?

\section{Maintainability}

Maintainability (ISO/IEC 9126-1, 2001) includes the analyzability, changeability, stability, and testability sub-characteristics. This set of characteristics reflects mainly the technical stakeholders' viewpoint, such as the developers and maintenance people (Hordijk \& Wieringa, 2005). Thus, the mobile-wireless features do not have significant influence upon them.

\section{Portability}

Portability (ISO/IEC 9126-1, 2001) includes the adaptability, installability, co-existence, and replaceability sub-characteristics. Portability is very important when the system is targeted to wide audience because of the large diversity of devices held by users and the lack of control over these devices, e.g. configuration devices and applications run by them. The application must adapt itself to the device features, both during installation and operation, and according to the user's preferences. The application installation on the device must be invisible to laypersons. The application operation in the mobile device needs to exist harmonically, sharing common resources, with other applications installed in the same device, especially co-existing with the telephone facilities.

- Does the application adapt to different devices?

- Does the application utilize middleware to adapt outputs to users and devices according to the user profile?

- Is the application easy to install in all required devices?

- Does the application interfere with other applications/services installed in the same device?

\section{Quality in Use}

Quality in use is the user's view of quality: "The capability of the software product to enable specified users to achieve specified goals with effectiveness, productivity, safety and satisfaction in specified contexts of use" (ISO/IEC 9126-4, 2004). Since mobile applications may be used while driving or walking, tasks which need the user attention, the application manipulation must be simple and intuitive, enforcing safety.

- Does the application prevent unnecessary paging for input or output?

- Does input operation require minimum typing? 


\section{Definition of the New Metrics}

In order to define the new metrics, the architecture and protocols of the mobile-wireless information systems were analyzed (Asunmaa et al., 2002; Green, 2003; Huber, 2004; Tarasewich, 2003; Varshney \& Vetter, 2002; Vaughan-Nichols, 2004) and the objects which affect the characteristics and sub-characteristics of quality were detected. As described in Figure 2, the method used to define the metrics is as following. Each identified object was decomposed into measurable attributes (ISO/IEC 15939, 2002). Each feature was assigned a unit measure and a scale, according to its meaning. This was done based on Kitchenham, Pfleeger, and Fenton methodology (1995). Using the objects identified and their attributes with corresponding units and scales, metrics (ISO/IEC 15939, 2002) were defined. The metrics are methods for the measuring process with specific formulas.

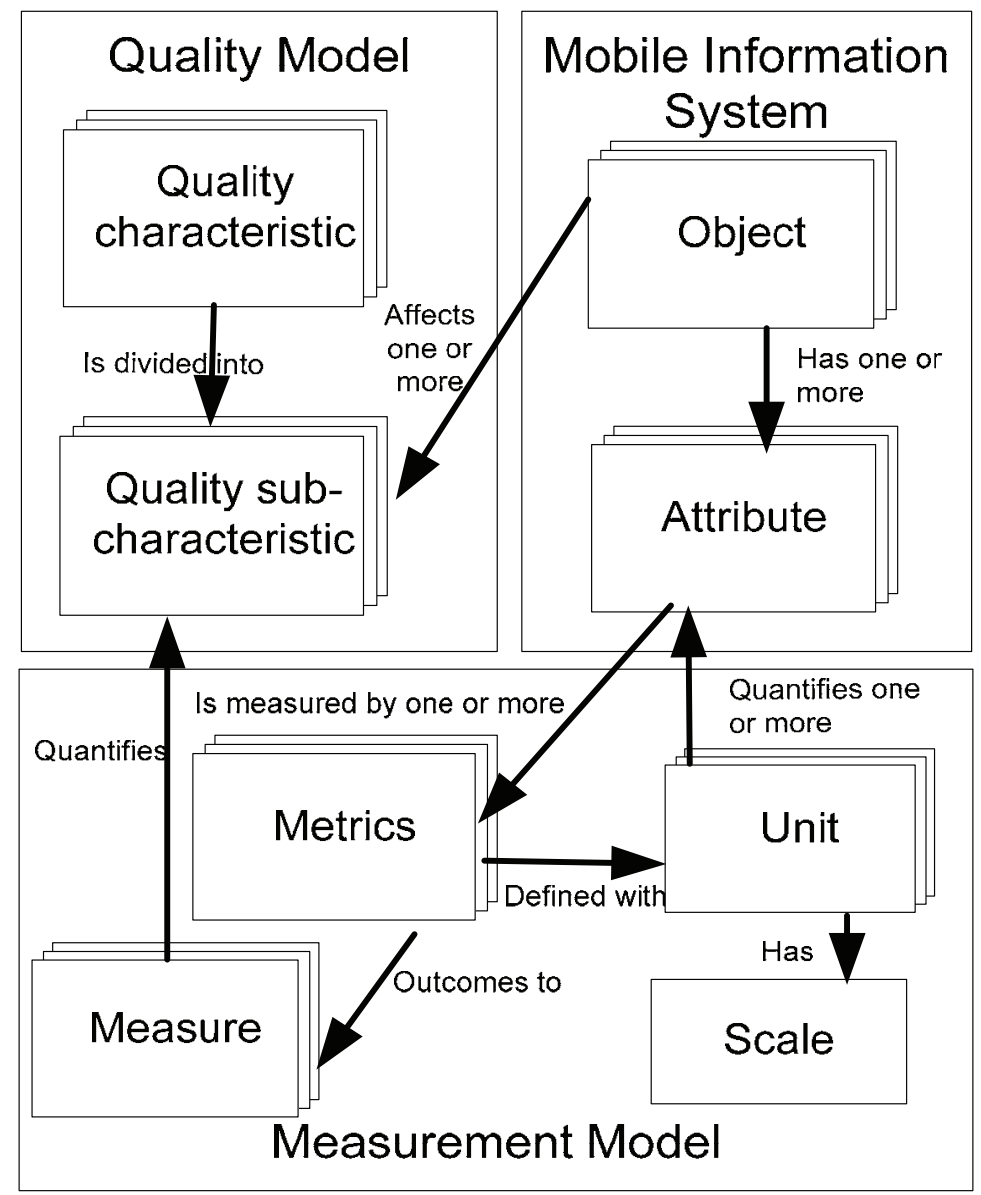

Figure 2 - Definition of metrics

The new defined metrics can be mapped in two different viewpoints:

- As a matrix which displays the metrics according to the specific problems in mobile-wireless information systems. Table 1 presents this viewpoint mapping.

- As a hierarchy starting from the quality characteristics, describing which metrics quantify the degree of quality for each characteristic and sub-characteristic. This is shown in Figure 3. 
Table 1 - Metrics mapped to mobile-wireless problems

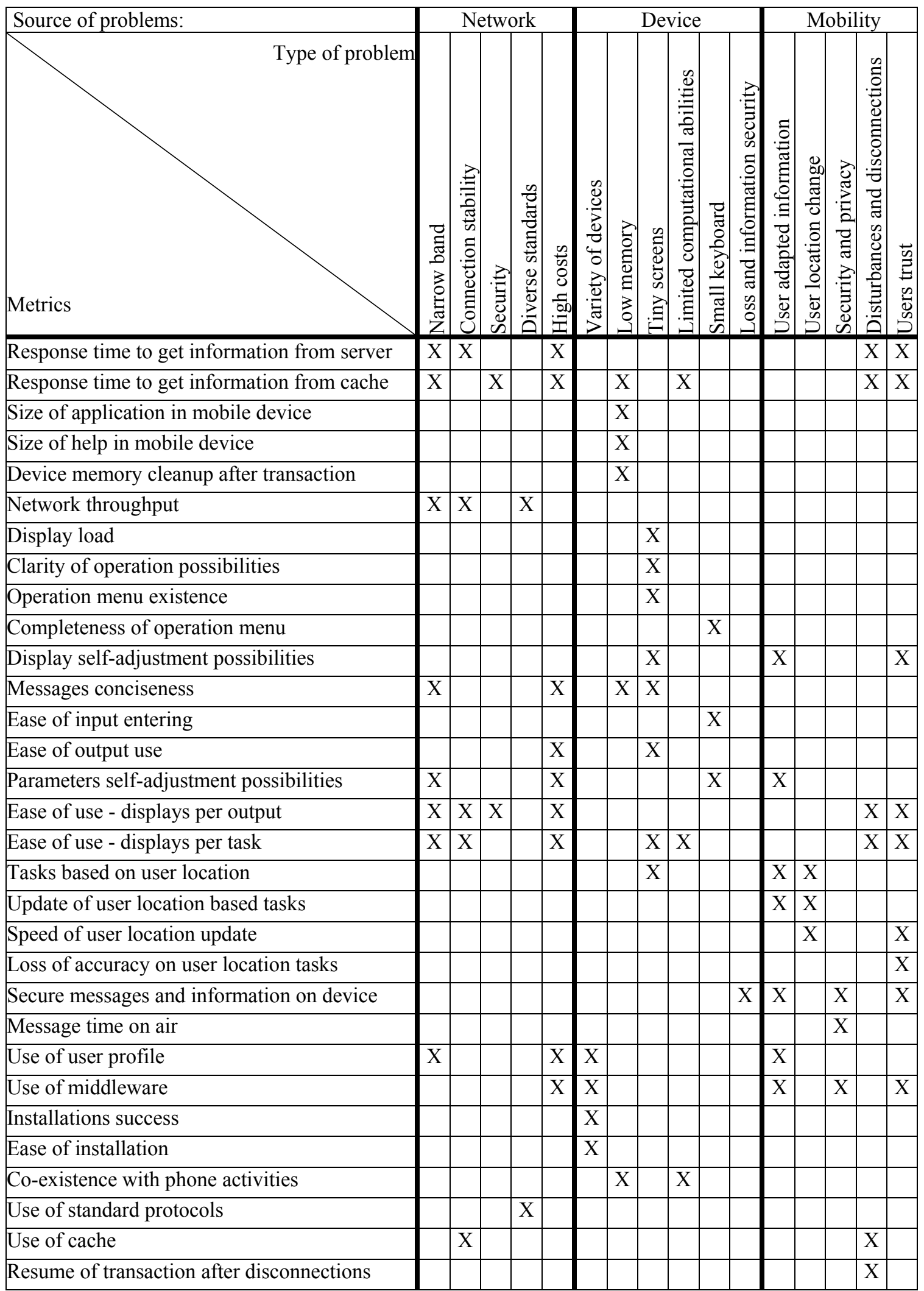




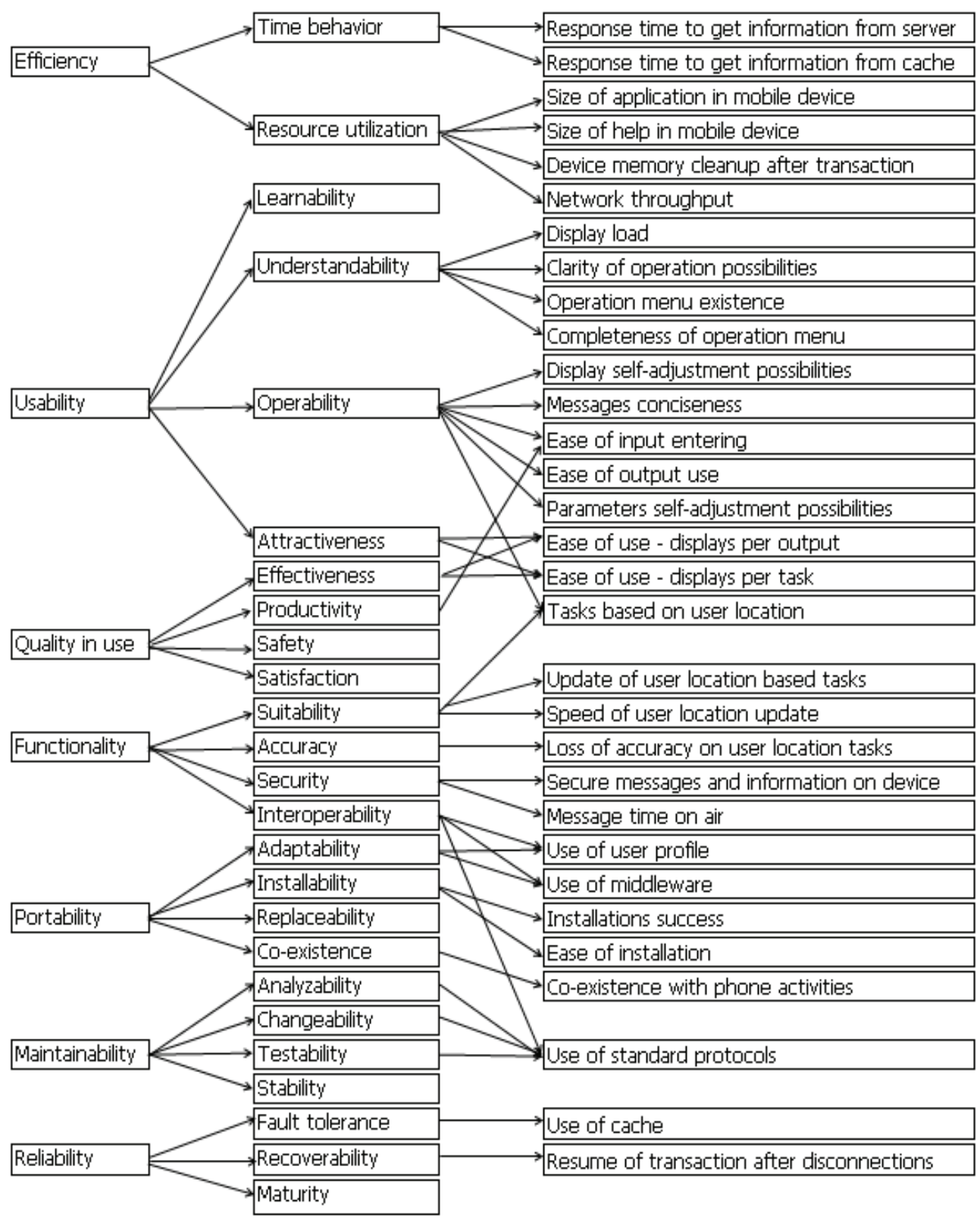

Figure 3 - Metrics mapped to quality characteristics

Tables 2 and 3 present examples of two new metrics developed in the research: "Display load" metric and "Memory cleanup" metric. 
Table 2 - Definition of "Display load" metric

\begin{tabular}{|c|c|}
\hline Metric Name & Display load \\
\hline Quality sub-characteristic & Usability - Understandability \\
\hline Purpose of the metric & $\begin{array}{l}\text { To what degree are the displays loaded? } \\
\text { The screen of the devices is small, and the understandability is } \\
\text { lower when the display is overloaded. }\end{array}$ \\
\hline Method of application & $\begin{array}{l}\text { Check the ratio between display size (information displayed) } \\
\text { and screen size. The ratios are categorized and given a score, } \\
\text { in inverse relationship to the display size, thus smaller dis- } \\
\text { plays categories receive greater scores. Then a weighted av- } \\
\text { erage is calculated. }\end{array}$ \\
\hline Metric type & Internal \\
\hline Attributes measured & $\begin{array}{l}\text { ScrS - Device screen size } \\
\text { PgS - Display size } \\
\text { DisNum - Number of displays in the system } \\
\text { i - Display number (1..DisNum) }\end{array}$ \\
\hline Computation & $\begin{array}{l}\text { Definition of categories and scores: } \\
X i=\left\{\begin{array}{lll}1 & \text { if } & 0<\frac{P g S_{i}}{S c r S} \leq 1 / 4 \\
2 / 3 & \text { if } & 1 / 4<\frac{P g S_{i}}{S c r S} \leq 1 / 2 \\
1 / 3 & \text { if } \quad & 1 / 2<\frac{P g S_{i}}{S c r S} \leq 3 / 4 \\
0 & \text { if } \quad 3 / 4 & <\frac{P g S_{i}}{S c r S} \leq 1\end{array}\right. \\
X=\frac{\sum_{i=1}^{D i s N u m} X_{i}}{D i s N u m} \\
\text { Where } \text { DisNum } \geq 1, S c r S \geq 1 \text { and } P g S_{i} \leq \text { DisS for all i. } \\
\text { If the size of the display is greater than the size of device } \\
\text { screen, the display will be partitioned according to the number } \\
\text { of pages to be displayed, and the calculation will be per- } \\
\text { formed for each one of these pages. }\end{array}$ \\
\hline Expected output & $0 \leq X \leq 1$ \\
\hline Interpretation of measured value & The higher the better \\
\hline Metric scale type & Ordinal \\
\hline Measure type & Count/Count \\
\hline Input to measurement & $\begin{array}{l}\text { Requirements Specification } \\
\text { Design } \\
\text { Product Description (from device manufacturer) }\end{array}$ \\
\hline ISO/IEC12207 reference & Software Design \\
\hline Target audience & $\begin{array}{l}\text { Developer } \\
\text { Maintainer } \\
\text { Human Interface Designer }\end{array}$ \\
\hline
\end{tabular}


Table 3 - Definition of "Memory cleanup" metric

\begin{tabular}{|c|c|}
\hline Metric Name & Memory cleanup \\
\hline Quality sub-characteristic & Efficiency - Resource Utilization \\
\hline Purpose of the metrics & $\begin{array}{l}\text { To what degree the memory is cleaned-up after completing a } \\
\text { task? } \\
\text { The devices have small memory, so any "garbage" left after } \\
\text { completing a task may decrease the subsequent work per- } \\
\text { formance. }\end{array}$ \\
\hline Method of application & $\begin{array}{l}\text { Check the size of free memory before starting the test, and } \\
\text { afterwards. }\end{array}$ \\
\hline Metric type & External \\
\hline Attributes measured & $\begin{array}{l}\mathrm{MmET}_{\mathrm{t}}-\text { free memory size at end of task i } \\
\text { ApS - Application Size } \\
\mathrm{MmS} \text { - Memory Size } \\
\text { TNum - Number of Tasks in System }\end{array}$ \\
\hline Computation & $\begin{array}{l}X=\frac{\sum_{t=1}^{T N u m} \frac{M m E T_{t}}{(M m S-A p S)}}{T N u m} \\
\text { where } \\
M m S-A p S>0 \\
\text { and TNum } \geq 1\end{array}$ \\
\hline Expected output & $0 \leq X \leq 1$ \\
\hline Interpretation of measured value & The higher the better \\
\hline Metric scale type & Ratio \\
\hline Measure type & Size \\
\hline Input to measurement & $\begin{array}{l}\text { User Monitoring Record } \\
\text { Test Report }\end{array}$ \\
\hline ISO/IEC12207 reference & $\begin{array}{l}\text { System Testing } \\
\text { Operational testing }\end{array}$ \\
\hline Target audience & $\begin{array}{l}\text { Requirer } \\
\text { Developer } \\
\text { Maintainer }\end{array}$ \\
\hline
\end{tabular}

\section{The Experiments}

Each metric defined by this research was validated theoretically and empirically at least by one of four different experiments performed in diverse technologies and devices, such as cellular phones and Personal Digital Assistants devices (examples of different displays can be seen in Figure 4):

1. The "PDA" experiment was performed on a system developed by Dooblo, a commercial company. The system allows conducting surveys on a PDA infrastructure. This system contains five different surveys, each including several displays.

2. The "Cellular" experiment was performed via a simulation developed specifically for this research. The system simulates a cellular phone application. This application allows technicians to service customers at home, per customers' complaints, that they generated via the cellular phone. This system was implemented twice, a "high quality" system and a "low quality" system, which enables comparisons. 
3. The "Where is the Child" experiment was performed using a service provided by a major mobile phone company, which enables parents to receive information about the location of their child over the cellular phone using GPS and cellular infrastructure.

4. The "Electronic Mail" experiment was performed in parallel in two different environments, in order to compare them, and on a third environment, not wireless and not mobile, as a reference point.

a. Electronic Mail on a PDA environment, based on Microsoft Outlook.

b. Electronic Mail on a cell phone, based on a service supplied by a major mobile phone company, which was developed based on Microsoft Outlook Express.

c. Electronic Mail on a desktop computer, based on Microsoft Outlook Express.

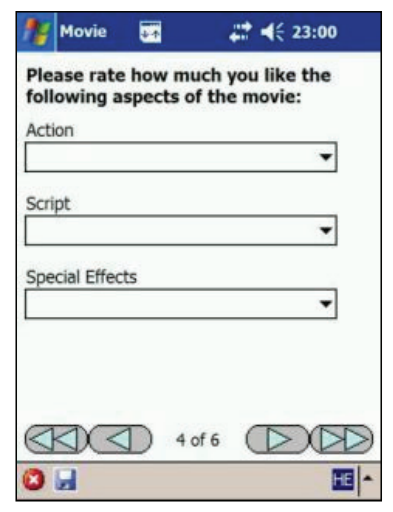

(1) "PDA"

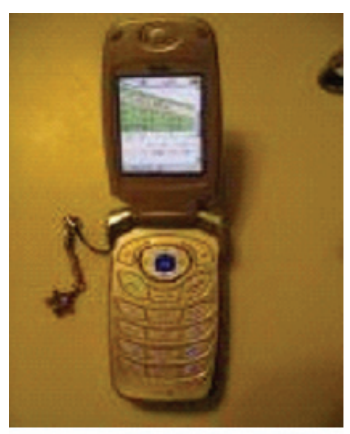

(3) "Where is the child?"

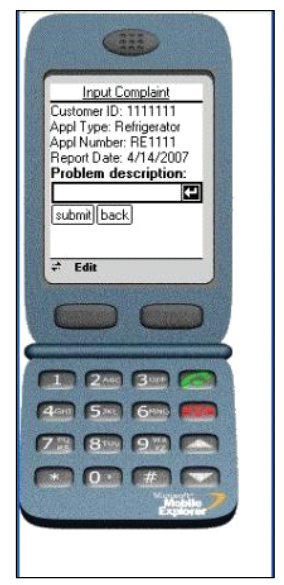

(2) "Cellular"

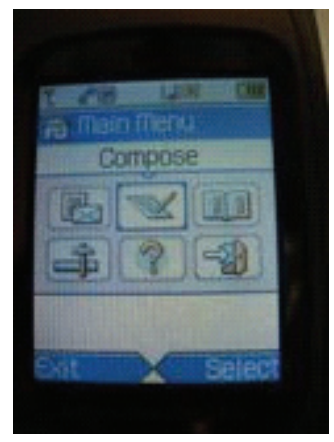

(4-b) "Electronic Mail"

Figure 4 - Examples of displays of the experiments

The aim of the validation was to prove that the metrics behave in a consistent and logical mode; thus, we can rely on them to quantify the quality of these systems, for example, by showing that the value of the metric grows when quality increases and vice versa.. 
The theoretical proof and the empirical experiments successfully validated the new metrics defined in this research. This paper focuses on the metrics development process, therefore the validation results are detailed only for the above example metrics.

The "Display Load" metric was validated in two different experiments, "PDA" and "Cellular". First, it was validated by the "PDA" experiment (Figure 5) which contains five different surveys, each including several displays. Therefore this experiment was further divided into six cases, one for each survey as it was an independent system and one for all together. For less loaded displays the metric value received was higher, i.e., the displays of the "Flight" survey which are the less loaded, got a metric value of 1, the highest possible. Second, the metric was validated using the "Cellular" experiment, once with the "High quality" system, and once with the "Low quality system", each was performed with empty and full displays (Figure 6).
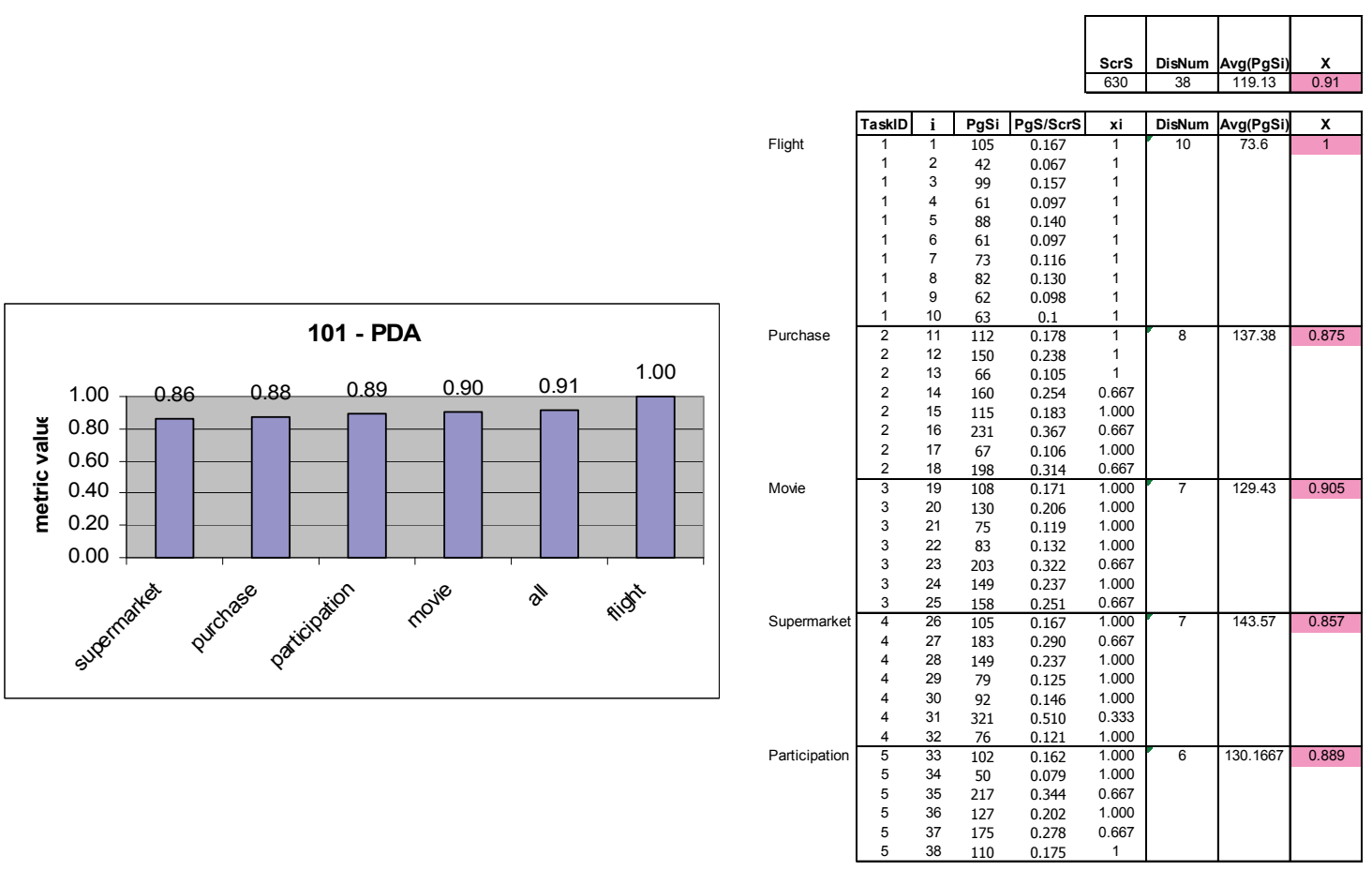

Figure 5 - Validation of "Display load" metric in "PDA" experiment

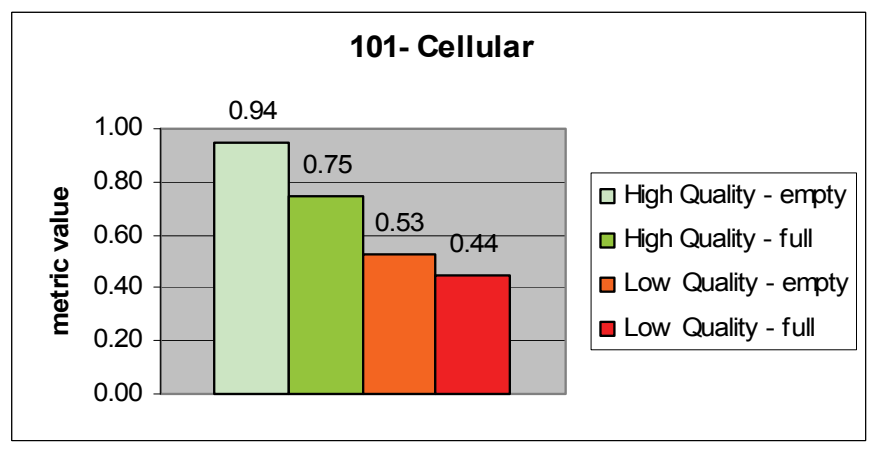

Figure 6 - Validation of "Display load" metric in "Cellular" experiment 
The "Memory Cleanup" metric was validated with the "PDA" experiment, for each survey, as it was an independent system and for all together. In the cases where the memory was better cleaned after completing the task, the metric value was higher, as shown in Figure 7.
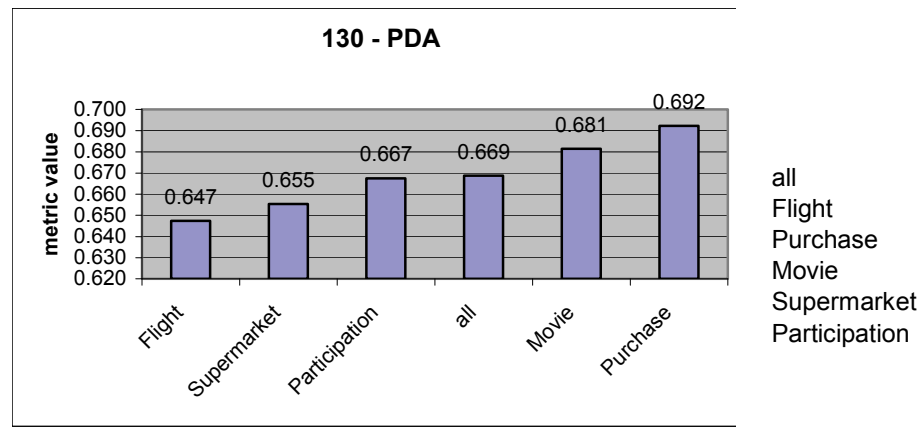

\begin{tabular}{|c|c|c|c|}
\hline Tnum & Aps & MmS & \\
\hline 150 & 5.75 & 35.49 & \\
\hline \multicolumn{4}{|c|}{} \\
\hline case & $\sum$ MmETi & & X \\
\hline all & 2983.45 & 100.318 & 0.669 \\
\hline 1 & 577.64 & 19.423 & 0.647 \\
\hline 2 & 617.62 & 20.767 & 0.692 \\
\hline 3 & 608.00 & 20.444 & 0.681 \\
\hline 4 & 584.76 & 19.662 & 0.655 \\
\hline 5 & 595.43 & 20.021 & 0.667 \\
\hline
\end{tabular}

Figure 7 - Validation "Memory Cleanup" metric in "PDA" experiment

\section{Conclusion}

This paper introduced two subjects and bound them together. First, it described mobile-wireless information systems and stated the new quality problems and challenges faced by these systems; second, it introduced the field of quality frames and standards for process and product quality assurance. Mobile-wireless information systems raise unique problems which affect quality. Therefore a set of questions was defined, which were developed methodologically to a list of metrics. These metrics enable objective quality evaluation and comparison of mobile-wireless information systems. The metrics were mapped in two different viewpoints, covering the quality characteristics on one hand, and the system problems on the other hand. A sample of two metrics was exhibited. The metrics listed above were developed according to the defined methodology and validated theoretically and empirically. Therefore, it confirms that these metrics enable objective quality evaluation and comparison of mobile-wireless information systems.

These metrics are useful when the quality of a mobile-wireless information system must be analyzed and quantified, for example when comparing two proposed systems, or when a system has to be developed or bought. When the metrics are used to compare systems, the higher the metric value, the higher the system's quality. However, when only one system has to be measured, the metrics need an external value to compare to. These values can be defined in advance according to the requirements of the system.

This research focused in mobile-wireless information systems which are activated through end devices which include a screen with displays, such as cellular phones and Personal Digital Assistance devices. The research can be expanded to new kinds of mobile-wireless information systems, emerging because of the rapid development of the technology and the wireless networks, such as wearable information systems and information systems based on RFID technology. 


\section{Acknowledgements}

I would like to express my appreciation and gratitude to my supervisors during my $\mathrm{PhD}$ studies: Dr. Moshe Mor, for his never ending support, his helpful comments and advice and Dr. David Schwartz for his productive remarks.

\section{References}

Asunmaa, P., Inkinen, S., Nykänen, P., Päivärinta, S., Sormunen, T., \& Suoknuuti, M. (2002). Introduction to mobile internet technical architecture. Wireless Personal Communications, 22, 253-259.

Basili, V. R., \& Rombach, H. D. (1988). The TAME project: Towards improvement-oriented software environments. IEEE Transactions on Software Engineering, 14(6), 758-773.

Calero, C., Ruiz, J., \& Piattini, M. (2004). A web metrics survey using WQM. Proceedings ICWE 2004, LNCS 3140, Springer-Verlag Heidelberg, 147-160.

Chrissis, M. B., Konrad, M., \& Shrum, S. (2006). CMMI: Guidelines for process integration and product improvement (2nd ed.). Boston, MA: Addison-Wesley.

Covella, G., \& Olsina, L. (2006). Assessing quality in use in a consistent way. Proceedings of the 6th International Conference on Web Engineering, ICWE'06, Palo Alto, California, USA, 1-8.

Di Pietro, R., \& Mancini, L. V. (2003). Security and privacy issues of handheld and wearable wireless devices. Communications of the ACM, 46(9), 74-79.

Green, R. (2003). Wap 2.0: What is it? Wap 2.0 is coming, sooner than you might think, and it's going to change everything. Wireless Business and Technology, 3(6), 34-38.

Herzberg, A. (2003). Payments and banking with mobile personal devices. Communications of the ACM, $46(5), 53-58$.

Hordijk, W., \& Wieringa, R. (2005). Surveying the factors that influence maintainability. Proceedings of the 10th European software engineering conference held jointly with 13th ACM SIGSOFT international symposium on Foundations of software engineering ESECFSE'05, Lisbon, Portugal, 385-388.

Huber, J. F. (2004). Mobile next-generation networks. IEEE Multimedia, 11(1), 72-83.

ISO/IEC 15939. (2002). Software engineering - Software measurement process. Geneva, Switzerland: International Organization for Standardization.

ISO/IEC 15504. (2004). Information technology - Process assessment (Parts 1-4). Geneva, Switzerland: International Organization for Standardization.

ISO/IEC 15504. (2006). Information technology - Process assessment (Part 5). Geneva, Switzerland: International Organization for Standardization.

ISO9000. (2000). Quality management systems - Requirements. Geneva, Switzerland: International Organization for Standardization.

ISO90003. (2004). Software engineering - Guidelines for the application of ISO 9001:2000 to computer software. [Previously: ISO9000-3 (1991)]. Geneva, Switzerland: International Organization for Standardization.

ISO/IEC 9126. (2001). Software engineering - Product quality - Part 1: Quality model. Geneva, Switzerland: International Organization for Standardization.

ISO/IEC 9126. (2004). Software engineering - Product quality - Part 4: Quality in use metrics. Geneva, Switzerland: International Organization for Standardization.

Lau, J. (2006). The state of European enterprise mobility in 2006. Forrester Research.

Lee, P. (2003). Mobile data comes of age: Wireless is big, we know that, but how big? Wireless Business and Technology, 3(9), 16-18. 
Kan, S. (2002). Metrics and models in software quality engineering (2nd ed.). Addison-Wesley.

Kitchenham, B., Pfleeger, S. L., \& Fenton, N. (1995). Towards a framework for software measurement validation. IEEE Transactions on Software Engineering, 21(12), 929-944.

Malladi, R., \& Agrawal, D. (2002). Current and future applications of mobile and wireless networks. Communications of the ACM, 45(10), 144-146.

Pandian, C. R. (2004). Software metrics: A guide to planning, analysis, and application. Auerbach Publications.

Schiller, J. (2000). Mobile communications. Addison-Wesley.

Stafford, T. F., \& Gillenson, M. L. (2003). Mobile commerce: What it is and what it could be. Communications of the $A C M, 46(12), 33-34$.

Spriestersbach, A. \& Springer, T. (2004). Quality attributes in mobile web application development. LNCS 3009, Proceedings of PROFES, Berlin: Springer-Verlag, 120-130.

Tarasewich, P. (2003). Designing mobile commerce applications. Communications of the ACM, 46(12), 5760.

Terho, M. (2002). Mobile web services and software quality. LNCS 2349, Proceedings of ESCQ, Berlin: Springer-Verlag, 2-6.

Terrenghi, L., Kronen, M., \& Valle, C. (2005). Usability requirements for mobile service scenarios. Proceeding of HCI International Conference, Las Vegas, USA, 1-10.

Varshney, U., \& Vetter, R. (2002). Mobile commerce: Framework, applications and networking support. Mobile Networks and Applications, 7, 185-198.

Vaughan-Nichols, S. J. (2004). Wireless middleware: Glue for the mobile infrastructure. IEEE Computer, 37(5), 18- 20 .

\section{Biography}

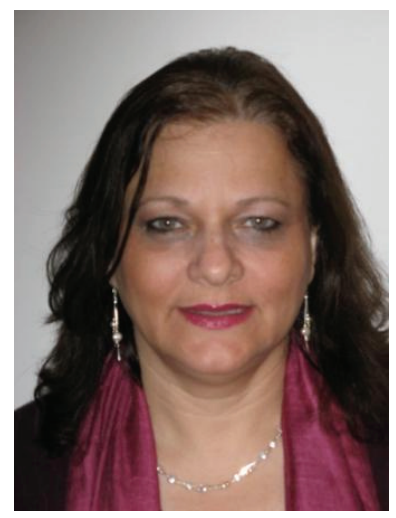

Dr. Ruti Gafni holds a PhD from Bar-Ilan University, Israel in the Business Administration School, focusing in Information Systems. She holds an M.Sc from Tel Aviv University and a BA (Cum Laude) in Economics and Computer Science from Bar-Ilan. She has more than 30 years of practical experience as project manager and analyst of information systems. She teaches in the Management and Economics MBA program, both at the Open University of Israel and at Netanya Academic College. 